\title{
Die ökonomische Dimension
}

\author{
Die Enquete-Kommission "Schułz des Menschen und der Umwelt" plädiert für \\ eine Gleichrangigkeit der Dimensionen Ökologie, Ökonomie und Soziales der \\ Nachhaltigen Entwicklung. Analog zu den bereits bekannten ökologischen Nach- \\ haltigkeitsregeln schlägt sie daher auch vier ökonomische Regeln vor. Wir \\ dokumentieren ihre Position in Auszügen:
}

(...) Nachhaltig zukunftsverträgliche Entwicklung reicht weit ïber Umweltfragen hinaus. (...) Als Ausweg zumindest aus dem [bestehenden] Definitionsdilemma bietet sich an, nachhaltig zukunftsverträgliche Entwicklung ähnlich wie

die positiven und offenen Begriffe Freiheit oder Gerechtigkeit als ,regulative Idee" $\mathrm{zu}$ verstehen, für die es nur vorläufige und hypothetische Zwischenbestimmungen geben kann. Es ergibt sich nämlich nicht nur das Problem, daß die gesellschaftlichen Vorstellungen von nachhaltig zukunftsverträglicher Entwicklung sowohl zeit-, situations- als auch kultur- und wissensabhängig sind. Darïber hinaus hängen die mit dem Leitbild verbundenen Problemempfindungen und politischen Schwerpunktsetzungen vom jeweiligen gesellschaftlichen und wirtschaftlichen Entwicklungsstand $a b$. Eine für alle Gesellschaften verbindliche Definition scheint deshalb ohne Aussicht auf Erfolg. Folglich kann auch nicht vorgegeben oder definiert werden, wie eine nachhaltig zukunftsverträgliche Gesellschaft oder eine nachhaltige Wirtschaft konkret auszusehen hat. (...)

\section{Die drei Dimensionen}

Im Grundsatz gehen [frühere] Überlegungen davon aus, daß bei einer über einen bestimmten Grad hinausgehenden Belastung eines ökologischen Systems Einschränkungen seiner Leistungsfähigkeit zu erwarten sind - möglicher- weise sogar der Zusammenbruch mit entsprechenden Folgen für die nachfolgenden Generationen. In jüngster Zeit (...) setzt sich zunehmend die Erkenntnis durch, daß auch im Bereich ökonomischer und sozialer Ordnungen Grenzen der Belastungsfähigkeiten existieren, die bei Überbeanspruchung $\mathrm{zu}$ ähnlichen Konsequenzen fuihren können. (...) Selbst wenn man (...) ein Primat der Umweltpolitik ableiten würde: der Sache wäre damit letztlich jedoch nicht gedient. Eine ökologisch dominierte Nachhaltigkeitspolitik wird im gesellschaftlichen Abwägungsprozeß immer dann unterliegen, wenn sich andere Problemlagen als unmittelbarer, spürbarer und virulenter erweisen und damit auch für politisches Handeln dringlicher und attraktiver sind. Selbst wenn sie sich durchsetzen kann, bleibt sie ohne Wirkung, denn letzlich dürfte nur eine Politik der Integration der drei Dimensionen in der Lage sein, die konzeptionelle Schwäche einer von wirtschaftlichen und sozialen Fragestellungen isolierten Umweltdiskussion zu überwinden. (...)Aufgrund der komplexen Zusammenhänge zwischen den drei Dimensionen bzw. Sichtweisen von Ökologie, Ökonomie und Sozialem müssen sie integrativ behandelt werden. Dabei geht es - bildhaft gesprochen - nicht um die Zusammenfuihrung dreier nebeneinander stehender Säulen, sondern um die Entwicklung einer dreidimensionalen Perspektive aus der Erfahrungswirklichkeit. Die Diskussion tendiert dahin, Nachhaltigkeitspolitik als Gesellschaftspolitik zu interpretieren, die im Prinzip und auf lange Sicht alle genannten Dimensionen gleichberechtigt und gleichwertig behandelt.

Im Kontext der Nachhaltigkeitsdiskussion geht es im ökonomischen und sozialen Sinne jedoch weniger um numerische und quantifizierte normative Zielsetzungen, als eher um qualitative Zielvorstellungen, die in ihrer Gesamtheit auf die Erhaltung der ökonomischen und sozialen Stabilität von Entwicklungsprozessen einerseits sowie die Aufrechterhaltung ihrer dynamischen und damit innovativen Funktionen andererseits ausgerichtet sind. Zentrales Ziel des Nachhaltigkeitsanliegens ist die Sicherstellung und Verbesserung ökologischer, ökonomischer und sozialer Leistungsfähigkeiten. Diese bedingen einander und können nicht teiloptimiert werden, ohne Entwicklungsprozesse als Ganzes in Frage zu stellen. So ist die Herstellung von Gerechtigkeit oder Chancengleichheit aus primär sozialpolitischem Interesse nicht allein ein soziales Ziel, sondern auch Voraussetzung für langfristige ökonomische Leistungsfähigkeit und folglich auch ein ökonomisches Ziel. Auch ökologische Ziele können kaum umgesetzt werden, wenn es Menschen aufgrund ihrer materiellen Bedingungen schwer gemacht wird, Rücksicht auf ökologische Ziele zu nehmen. Ähnliche Überlegungen ergeben sich auch in umgekehrter Ziel-Mittel-Zweck-Relation. (...)

Die Notwendigkeit, zur Umsetzung der Nachhaltigkeit alle drei Dimensionen der gesellschaftlichen Wirklichkeit zu beachten, ließ es der Kommission sinnvoll erscheinen, neben den ökologischen Grundregeln auch ökonomische und soziale Regeln zu formulieren. Die im folgenden beschriebenen Überlegungen der Enquete-Kommission zur ökonomischen und sozialen Bedingung für eine nachhaltig zukunftsverträgliche Entwicklung sind ebenso wie die formulierten ökonomischen und sozialen Regeln weit weniger ausgereift als die ökologischen Regeln. Denn die Kommission mußte feststellen, daß bisher weder ausreichende wissenschaftliche Grundlagen für dieses Vorhaben zur Verfügung stehen noch eine ausfiuhrliche, politische Debatte geführt werden konnte. (...) Die Kommission versteht ihre Ausführungen deshalb als einen ersten Entwurf. Sie ist sich der Vorläufigkeit und Skizzenhaftigkeit der Thesen bewußt. Gerade deshalb erhofft sie sich, Diskussionen provozieren und die wissenschaftliche und politische Auseinandersetzung mit dem Thema anregen zu können. (...)

\section{Ökonomische Ziele}

Die Bundesrepublik Deutschland verdankt ihre wirtschaftliche Entwicklung einer liberalen, sozialen Wirtschaftsordnung, in der Elemente

\section{Die Enquete-Kommission.}

"Schutz des Menschen und der Umwelt - Ziele und Rahmenbedingungen einer nachhaltig zukunftsverträglichen Entwicklung" wurde vom 13. Deutschen Bundestag am 1.Juni 1995 eingesetzt. Sie knüpfte an die Arbeit einer Vorläufer-Enquete in der 12. Legislaturperiode an und hat ihren Abschlufbericht am 22. Juni 1998 vorgelegt (vgl. Ökologisches Wirtschaften $5 / 98$, S. 6). Ihr gehörten unter dem Vorsitz von Ernst Schwanhold bzw. Marion Cospers-Merk (SPD) 12 Bundestagsabgeordnete entsprechend der Stärke der dort vertretenen Fraktionen bzw. Gruppen und 12 von diesen benannte Sachverständige an. Die Sachverständigen kamen aus Wirtschaftsverbänden, Gewerkschaft und insbesondere der Wissenschaft. Enquete-Kommissionen werden vom Deutschen Bundes. tag eingesetzt, um Zukunftsfragen von übergeordneter Bedeutung abseits der Togespolitik mit Hilfe externer Sachverständiger zu erörtern und politische Entscheidungen vorzubereiten. 
zentraler Steuerung zugunsten dezentraler Organisation der Wirtschaftsprozesse zurücktreten. Das Leitbild für diese Wirtschaft, die „soziale Marktwirtschaft", ist in der Gesellschaft tief verankert. Die Legitimität dieser Wirtschaftsordnung mit ihren grundlegenden Werten, Regeln und Normen als Basis des wirtschaftlichen Lebens gilt als gesichert. (...)

Es ist notwendig, die Ziele, die seit langem bekannt sind, und die im Rahmen der sozialen Marktwirtschaft angestrebt werden sollen, ernsthafter und konsequenter $\mathrm{zu}$ verfolgen, um sowohl die Markt- und Wettbewerbsbedingungen als auch die soziale Akzeptanz dieser Ordnung zu erhalten und zu verbessern. Es scheint auch notwendig zu sein, die Rückbesinnung auf grundlegende Gesetze der Ordnungspolitik (z.B. Gesetz gegen Wettbewerbsbeschränkungen) und auf das grundlegende Gesetz der Prozeßpolitik (Stabilitäts- und Wachstumsgesetz) einzufordern. Die dynamischen Wettbewerbsfunktionen müssen vor allem im Hinblick auf eine zunehmende Wettbewerbsintensität im europäischen Binnenmarkt gestärkt werden.

Hinter diesen grundlegenden Gesetzen stehen in der sozialen Marktwirtschaft Ziele wie Frieden, Freiheit, Gerechtigkeit, Sicherheit und Wohlstand. Marktwirtschaft ist nicht Selbstzweck. Sie muß im Dienst der Bedürfnisse des Menschen stehen. (...) Die grundsätzliche Ausrichtung gesamtwirtschaftlicher Zielsetzungen ist damit die Schaffung und Erhaltung der Effizienz von Interaktionsprozessen. Die in den meisten Industrieländern festgelegte Markt- und Wettbewerbsordnung ist letztlich grundsätzlich darauf angelegt, die Herausbildung von Preisen einem durch Markt und Wettbewerb institutionalisierten Verfahren zu überlassen. Die der regulativen Idee der Nachhaltigkeit innewohnende Vorstellung eines Such- und Lernprozesses findet im Wettbewerb der Marktwirtschaft ihre ökonomische Ausprägung. (...)

Die Erhaltung und nachhaltige Sicherung der Wettbewerbs- und Marktfunktionen ist somit ein unverzichtbares Zwischenziel zur Erreichung gesellschaftlicher Ziele, denen die Wirtschaft zu dienen hat. Unter dieser Prämisse gilt es, ökonomische Qualitätsziele zu beschreiben. Ökonomische Qualitätsziele sollen langfristig angestrebte, am Leitbild der nachhaltig zukunftsverträglichen Entwicklung und damit am Ziel der Erhaltung der Funktionsfähigkeit der ökonomischen Systeme orientierte Eigenschaften beschreiben. (...) Ökonomische Handlungsziele geben Schritte an, die notwendig sind, um die in ökonomischen Qualitätszielen beschriebenen Zustände und Eigenschaften der ökonomischen Dimension der Nachhaltigkeit zu erreichen. (...) Handlungsziele orientieren sich primär an den Rahmenbedingungen, die über die Qualität der Wettbewerbsund Marktprozesse entscheiden. Eine weitere Operationalisierung und Quantifizierung ökonomischer Ziele kann nur vor dem Hintergrund der nachfolgend dargestellten ökonomischen Handlungsprinzipien der Nachhaltigkeit (,grundlegende Regeln") sowie einer konkret vorliegenden Problemkonstellation erfolgen. (...)

\section{Ökonomische Handlungsmaximen}

Wirtschaften hat die übergeordnete Funktion, knappe Güter mit möglichst geringen Kosten der Verwendung mit der höchsten Wertschätzung zukommen zu lassen: So sollen die verfügbaren Ressourcen an Arbeitskraft und natürlicher Produktivität so eingesetzt werden, daß eine bestmögliche Versorgung der Bevölkerung mit Gütern und Dienstleistungen erreicht wird. Alle vorhandenen Produktionsfaktoren sollen ihrer produktivsten Verwendung zugefuihrt werden.

Konstituierend für das System der sozialen Marktwirtschaft ist das dezentrale Entscheidungssystem der Märkte mit dem Wettbewerb als Motor wirtschaftlicher Entwicklung (...). Gleichwohl hoben deren geistigen Väter den unlösbaren Zusammenhang zwischen freien Märkten und einer funktionsfähigen Rahmenordnung hervor, die den Wettbewerb in gesellschaftlich erwünschte Bahnen lenken soll. Damit marktwirtschaftliche Ordnung auf Dauer erhalten werden können, müssen ,,selbstzerstörerische" Kräfte neutralisiert werden. (...) Die Vernachlässigung sozialer und ökologischer Belange ist eine mögliche unerwünschte Nebenwirkung der Vorteile, die mit einem freien Wettbewerb einhergehen. Auch hier sind Korrekturen durch entsprechende Interventionen des Staates notwendige Bedingung für die Erhaltung der Verhältnisse für alles Wirtschaften. Was auf den ersten Blick wie eine Einschränkung ökonomischer Freiheiten anmutet, ist auf lange Sicht die nachhaltige Sicherung freier Marktprozesse. Insofern ist auch aus „puristisch“ ökonomischer Sicht die ökologische und soziale Marktwirtschaft eine Notwendigkeit, um moderne Marktwirtschaften überlebensfähig zu machen. (...)

\section{Vier Regeln}

Vor diesem Hintergrund schlägt die EnqueteKommission als Anregung für die weitere Diskussion in und mit den entsprechenden Fachgebieten nachstehende, noch nicht anschließend diskutierte Regeln vor, die aus ökonomischer Sicht der Nachhaltigkeit beachtet werden sollten (vgl. Kasten).

Vordringliches Ziel dieser Handlungsanleitungen ist die Minderung von Knappheiten, der sich die Menschen ausgesetzt sehen, sowie die Erhöhung des Wohlstandes. Die ökonomische Dimension, verstanden als durch Menschen gesetzte Rahmenordnung, kennt ökonomische Ziele wie statische oder dynamische Effizienz nicht als Selbstzweck, da hinter diesen Zielen immer soziale und ökologische Ziele stehen, die unter dem Begriff des Gemeinwohls subsumiert werden können. Vor diesem Hintergrund wird die erste Regel verständlich, die darauf abzielt, die Rahmenordnung so zu gestalten, daß die eigensüchtigen Triebkräfte der Menschen in geeigneter Weise auch der Allgemeinheit nützlich gemacht werden.

Die zweite und dritte Regel betonen die Funktionen von Markt und Wettbewerb (,invisible hand") für die Überwindung von Knappheit vor

Ökonomische Nachhaltigkeitsregeln

1. Das ökonomische System soll individuelle und gesellschaftliche Bedürnisse effizient befriedigen. Dafür ist die Wirtschaftsordnung so zu gestalten, daß sie die persönliche Initiotive fördert (Eigenverantwortung) und das Eigeninteresse in den Dienst des Gemeinwohls stellt (Regelverantwortung), um das Wohlergehen der derzeitigen und künftigen Bevölkerung zu sichern. Es soll so organisiert werden, daß es ouch gleichzeitig die übergeordneten Interessen wahrt.

2. Preise müssen daverhaft die wesentliche Lenkungsfunktion ouf Märkten wahrnehmen. Sie sollen dazu weitestgehend die Knappheit der Ressourcen, Senken, Produktionsfaktoren, Güter und Dienstleistungen wiedergeben.

3. Die Rohmenbedingungen des Wettbewerbs sind so zU gestalten, daß funktionsfähige Märkte entstehen und aufrechterhalten bleiben, Innovationen angeregt werden, daß langfristige Orientierung sich lohnt und der gesellschaftliche Wandel, der zur Anpassung an zukünftige Erfordernisse nötig ist, gefördert wird.

4. Die ökonomische Leistungsfähigkeit einer Gesellschaft und ihr Produktiv-, Sozial- und Humankapital müssen im Zeitlouf zumindest erhalten werden. Sie sollten nicht bloß quantitativ vermehrt, sondern vor allem auch qualitativ ständig verbessert werden. 
dem Hintergrund vielfältig unterschiedlicher individueller Bewertungen des Nutzen bzw. Schadens, des Risikos und der Chancen, die mit Gütern, Dienstleistungen, Lebenssituationen usw. verbunden sind.

Die letzte Regel zielt auf die Erweiterung des Möglichkeitsraumes zur Überwindung von Knappheiten für nachfolgende Generationen. Die Forderung nach Erhalt des Produktiv-, Sozial- und Humankapitals beinhaltet hierbei auch den Erhalt der Ertragskraft eines sich in seiner Zusammensetzung stets wandelnden Kapitalbestandes.

(...) Mit den vier grundlegenden Regeln zum dauerhaften Umgang mit natürlichen Ressourcen hat die Enquete-Kommission in der 12. Legislaturperiode die Begrenztheit der Entnahme- und Regenerationsfähigkeit des Naturhaushaltes in den Vordergrund gerückt. (...) [Dem] ist eine fünfte Regel zum Schutz der menschlichen Gesundheit hinzugefügt worden. Die ökonomischen und sozialen Regeln ergänzen diese Regeln im Sinne von Verhaltensweisen als Grundlage einer integrativen Politik zur Sicherung einer zukunftsfähigen gesellschaftlichen Entwicklung. (...)

Gesellschaftliche Zielsetzungen müssen im Zeitablauf immer wieder aufs neue an die veränderten Realitäten angepaßt werden. Notwendig dazu sind verbindliche Etappenziele. Durch verläßliche Zielvorgaben sollen die Anstrengungen der Akteure die gewünschte Richtung einschlagen. Sollten positive Ziele weder vorliegen noch formulierbar sein, so lassen sich bestimmte Entscheidungen, Maßnahmen und Zustände zuweilen eindeutig als Verstöße gegen die Postulate der nachhaltig zukunftsverträglichen Entwicklungen identifizieren. Ziele müssen im Sinne der Idee der Nachhaltigkeit weiterentwickelt, ein ausgewogenes Maß an allgemein akzeptierten Leitplanken für die gesellschaftliche Entwicklung formuliert werden.

Der Text ist ein Auszug aus dem Kapitel 2 "Das Leitbild einer nachhaltig zukunftsverträglichen Entwicklung" des Abschlußberichts der Enquete-Kommission "Schutz des Menschen und der Umwelt - Ziele und Rahmenbedingungen einer nachhaltig zukunftsverträglichen Entwicklung": Konzept Nachhaltigkeit - Vom Leitbild zur Umsetzung. Bundestags-Drucksache 13/11200, S. 16-29. Wir danken der Enquete-Kommission für die freundliche Genehmigung.
Beschreiben die ökonomischen Regeln der Enquete-Kommission nachhaltiges Wirtschaften?

\section{Gefahr der Relativierung}

Die gleichrangige Behandlung der Dimensionen Ökologie, Ökonomie und Soziales übersieht die ökologischen Grenzen aller menschlichen Aktivitäłen und die daraus resultierende Abhängigkeit der anderen Dimensionen von der ökologischen Dimension der Nachhaltigkeit. Diese droht dabei ins Hintertreffenzu geraten. Deshalb werden alternative ökonomische Regeln vorgeschlagen.

B Von Jürgen Rochlitz edauerlicherweise hat sich die Mehrheit der Enquete-Kommission „Schutz des Menschen und der Umwelt" nicht auf eine klare Definition von Nachhaltigkeit einigen können. Auch zu Forderungen nach einschneidenden Veränderungen des ökonomischen Istzustandes zur Sicherung der natürlichen Lebensgrundlagen und einer angemessenen Beschäftigung konnte sie sich nicht entschließen. Sie wollte oder konnte nicht mehr in der Nachhaltigkeit sehen als eine regulative Idee.

So wird von der Kommissionsmehrheit nicht wahrgenommen, welche langfristig orientierte Klugheit und Ökonomie dem Leitbild der Nachhaltigkeit innewohnt. Damit verabschiedet sie sich nicht nur aus der internationalen Debatte um die Konkretisierung des Leitbildes, sondern auch von so manchen konkreten Vorstellungen aus der Vorgänger-Kommission (1).

Die seinerzeit dort formulierten ökologischen Nachhaltigkeitsregeln zum Erhalt des natïrlichen Realkapitals wurden insbesondere von Seiten der CDU-Mitglieder und deren Sachverständigen als revisionsbedürftig, weil $\mathrm{zu}$ einseitig, betrachtet. Diese ökologiekritische Haltung führte zur Formulierung von ökonomischen und sozialen Regeln, die allein der Relativierung der wissenschaftich anerkannten und begründbaren ökologischen Nachhaltigkeitsregeln dienen sollten. Die Kommission hat nicht die Gefahr erkannt, daß damit die wesentlichen Themen der Umweltpolitik auch künftig hinter denjenigen der Wirtschafts- und Sozialpolitik rangieren, sondern diese sogar noch verstärkt. Die gleichrangige Behandlung der Dimensionen Ökologie, Ökonomie und Sozialwesen übersieht sträflicherweise die von verschiedenen Fachinstitutionen formulierten ökologischen Grenzen aller menschlichen Aktivitäten (2). Damit wird auch der Ausgangspunkt der internationalen Nachhaltigkeitsdebatte, die dringliche Warnung der Brundtland-Kommission (3), sträflich mißachtet.
Die konsequente Beachtung der Tragekapazität der Umwelt ist aber nichts anderes als ökologisch-soziale Langzeitökonomie zugunsten lebender und zukünftiger Generationen. Sie bedeutet zukunftsorientierte Klugheit mit den Elementen Vorsicht, Vorsorge und Behutsamkeit. Dem von der Kommission verfolgten Integratio nsmodell von Nachhaltigkeit konnte der Autor daher nicht folgen (4).

\section{Istzustand festgeschrieben}

Die Vorschläge zu den ökonomischen und sozialen Regeln für Nachhaltigkeit halten eher den Istzustand der bundesdeutschen Marktwirtschaft und damit den Zustand fehlender Nachhaltigkeit fest, als daß sie die nötige Basis liefern für eine grundsätzliche ökonomische Transformation. Zu den nötigen Veränderungen unserer Produktions- und Konsummuster sind keine Ansätze zu finden. Es fehlt jegliche Vision einer zukunftsfähigen nachhaltigen, damit auch ökologischen und sozialen Marktwirtschaft. Sogar eines der drückendsten Probleme unserer Zeit, nämlich die ausreichende Versorgung der Menschen mit Beschäftigung, wird in den Regeln der Kommissionsmehrheit mit keinem Wort erwähnt! Den Regeln fehlt zudem die nötige Klarheit und der Bezug zur Nachhaltigkeit, also eine Zukunftsorientierung, wie sie die ökologischen Regeln auszeichnet.

Weder die Sicherung des Wohlergehens der Bevölkerung, noch funktionsfähige Märkte, noch die Mehrung des Produktivkapitals, um nur diese Beispiele aus drei ökonomischen Regeln zu nennen, können neue und wesentliche Elemente für eine nachhaltige Entwicklung sein. Sie stellen Selbstverständlichkeiten dar oder stammen aus altbekannten Vorstellungen vom Wachstum. Zur Nachhaltigkeit fiihrt da schon eher, wenn Preise die Knappheit von Ressourcen und Senken wiedergeben sollen, wie es in der zweiten ökonomischen Regel gefordert wird. Auch die Anregung von Innovationen und die Belohnung langfristiger 
Orientierung (dritte ökonomische Regel) wird dieser Richtung gerecht. Mit den ökonomischen Regeln insgesamt wurde der Effizienz der Ökonomie die Rolle einer eigenständigen Zielkategorie zugebilligt, ohne zu berücksichtigen, daß ,alles Wirtschaften und auch die Wohlfahrt im klassischen Sinn unter dem Vorbehalt der ökologischen Nachhaltigkeit stehen“ (5).

\section{Stärkere Regeln nołwendig}

Ökonomische Nachhaltigkeitsregeln müssen berücksichtigen, daß die Natur ein Lebenserhaltungssystem höchster Komplexität ist, das die Grundvoraussetzung überhaupt jeglichen Wirtschaftens darstellt. Als Lieferantin für den ökonomischen Prozeß darf ihr nur soviel abgenommen werden, wie sie zu regenerieren vermag, sagen schon die ökologischen Regeln. Der ökonomische Prozeß darf jedoch nicht zu Lasten des Lebenserhaltungssystems optimiert werden, dies müssen die ökonomischen Regeln zum Ausdruck bringen. Ebenso wenig darf der ökonomische Prozeß zu Lasten künftiger Generationen optimiert werden, wenn man ihn zukunftsfähig gestalten will. Unter diesen Aspekten sind in meinem Sondervotum die folgenden ökonomischen Regeln vorgeschlagen worden: 1. Gewinne und materielle Zuwächse zu Lasten oder auf Kosten nachfolgender Generationen sind zu minimieren, schließlich gänzlich zu vermeiden.

2. Schulden der öffentlichen Hand müssen von der politisch verantwortlichen Generation abgetragen werden, es sei denn sie betreffen erwiesenermaßen nachbaltig zukunftsfäbige Investitionen zugunsten nachfolgender Generationen.
3. Die ökonomischen Rabmenbedingungen sind so zu gestalten, daß sie dem Ziel des Erhalts der natürlichen Lebensbedingungen, damit auch des Erhalts der ökonomischen Grundlagen, sowie der Förderung der Beschäftigung dienen.

Mit diesen Regeln wird die Effizienz der Ökonomie als Instrument begriffen, das den Zielen der Ökologie und des Sozialen dient, wenn eine nachhaltige Entwicklung angestrebt werden soll. Selbstverständlich bedeutet dies eine tiefgreifende Korrektur bisheriger Fortschritts- und Wachstumsvorstellungen, die sich in ihrer gegenwärtigen Form nicht länger als tragfähig erweisen (6).

Eine solche Transformation ist weder durch Verordnungen noch durch Bekehrungen möglich. Es bedarf dazu eines Wandels in Kultur und Bildung. Gut leben statt viel haben (Öko-Philosophie), Nutzung solarer Energie statt Ausbeutung fossiler Energieträger (Öko-Konsistenz), Kreislauf- statt Durchflußwirtschaft (Öko-Effizienz), Vermeidung und Verzicht bei unverträglichen Verhaltensweisen für Um- und Mitwelt (ÖkoSuffizienz) sind die großen Herausforderungen. Das hierzu nötige Umdenken kann nur gelingen, wenn in Bildung, Aus- und Fortbildung und im kulturellen Leben daran gearbeitet wird. Um dieser besonderen Bedeutung von Kultur und Bildung gerecht zu werden, wurden sie im Sondervotum als vierte Dimension der Nachhaltigkeit bezeichnet.

Mit den im Sondervotum formulierten Regeln wurde ein Konzept starker Nachhaltigkeit verfolgt. Deren Ergebnis wäre nicht ein Erhalt oder gar Anwachsen materiellen Wohlstands auf Kosten der Natur und zu Lasten kïnftiger Generationen oder der Entwicklungsländer, wie bisher. Vielmehr müßten wir uns zufrieden geben mit einem Wohlbefinden und Wohlergehen fïr alle in einer vor weiterer Zerstörung bewahrten Natur. Hierzu sind jedoch mit erheblichen Anstrengungen sämtliche Möglichkeiten und Instrumentarien unserer Gesellschaft zu aktivieren.

\section{Anmerkungen}

(1) Enquete-Kommission "Schutz des Menschen und der Umwelt “ des 12. Deutschen Bundestages: die Industriegesellschaft gestalten. Bundestags-Drucksache 12/8260.

(2) z.B. Rat von Sachverständigen für Umwellfragen: Umweltgutachten 1994 und 1996;

Unabhängige Sachverständigenkommission zum Umweltgesetzbuch: Umweltgesetzbuch, UGB-KomE, 1998.

(3) Volker Hauff (Hrsg.): Unsere gemeinsame Zukunft, Bericht der Weltkommission für Umwelt und Entwicklung. Greven 1987

(4) Vgl. das ausführliche Sondervotum zum Kapitel 2 des Enquete-Berichts. BT-Drs 13/11200, S. 216-228.

(5) Umweltbundesamt: Nachhaltiges Deutschland, Berlin 1997.

(6) Rat von Sachverständigen für Umwelffragen: Umweltgutachten 1994, Punkt $1 \mathrm{ff}$.

Der Autor
Prof. Dr. Jürgen Rochlitz war als Bundestags-
abgeordneter von Bündnis $90 /$ Die Grünen
Mitglied der Enquete-Kommission "Schuiz des
Menschen und der Umwelt".
Kontakt: Die Ecke 1, 35099 Burgwald.
Tel. $06547 / 89041$, Fax $06457 / 393$.

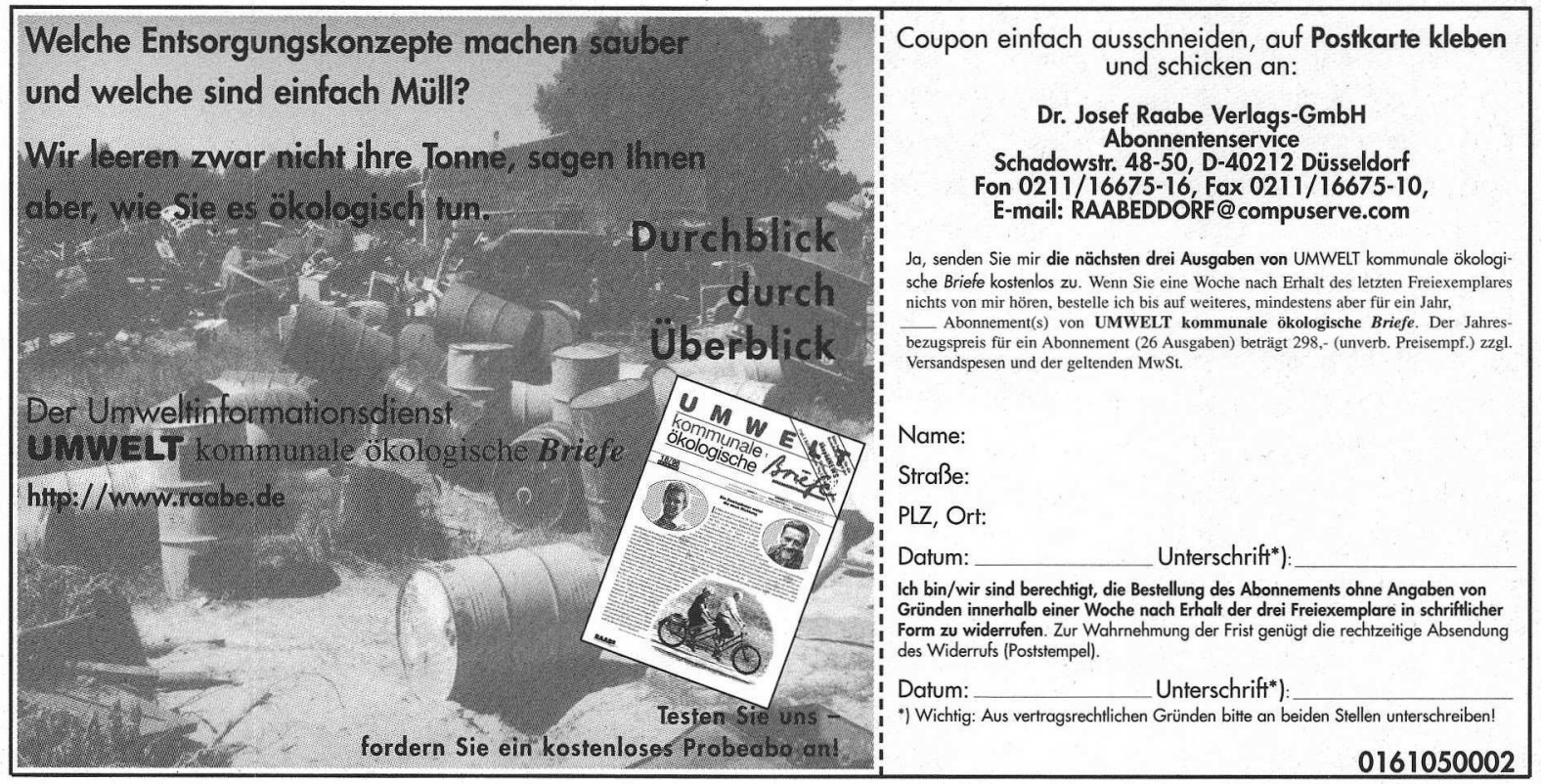


(c) 20I0 Authors; licensee IÖW and oekom verlag. This is an article distributed under the terms of the Creative Commons Attribution Non-Commercial No Derivates License (http://creativecommons.org/licenses/by-nc-nd/3.o/), which permits unrestricted use, distribution, and reproduction in any medium, provided the original work is properly cited. 